NISTIR 7796

\title{
Model Based Engineering Standardization and Validation
}

Joshua Lubell

Nathan W. Hartman

Doug Cheney

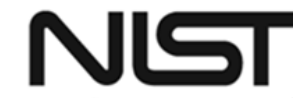

National Institute of Standards and Technology U.S. Department of Commerce 
NISTIR 7796

\title{
Model Based Engineering Standardization and Validation
}

\author{
Joshua Lubell \\ Engineering Laboratory \\ National Institute of Standards and Technology \\ Nathan W. Hartman \\ Purdue University \\ Doug Cheney \\ ITI Transcendata
}

July 6, 2011

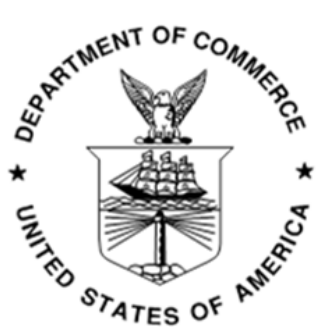

U.S. Department of Commerce Gary Locke, Secretary 


\section{MODEL BASED ENGINEERING STANDARDIZATION AND VALIDATION}

\author{
Joshua Lubell \\ National Institute of Standards \\ and Technology \\ Gaithersburg MD, USA \\ lubell@nist.gov
}

\author{
Nathan W. Hartman \\ Purdue University \\ West Lafayette IN, USA \\ nhartman@purdue.edu
}

\author{
Doug Cheney \\ ITI TranscenData \\ Milford OH, USA \\ Doug.Cheney@transcendata.com
}

\section{ABSTRACT}

3D Model-Based Engineering (MBE) is an approach to product design, manufacturing, and support where a digital three-dimensional representation of the product serves as the normative source for information communicated throughout the product's lifecycle and supply chain. MBE simplifies data management and provides a more powerful communication medium than 2D-based environments. Lightweight formats, offering a low-cost way for humans to view and potentially for applications to consume geometry and Product Manufacturing Information (PMI), are a critical component of $\mathrm{MBE}$ and enable collaboration without requiring that business partners buy expensive Computer-aided Design (CAD) systems. These formats are becoming increasingly popular - and increasingly complex - as their representational capabilities grow. Their value to industry is driving a push for standardization. We discuss the advent of MBE, the standards and technologies that make it possible, and the importance - and challenges - of product data validation.

\section{NOMENCLATURE}

$\begin{array}{ll}\text { ASME } & \text { American Society of Mechanical Engineers } \\ \text { CAD } & \text { Computer-aided Design } \\ \text { CAM } & \text { Computer-aided Manufacturing } \\ \text { GD\&T } & \text { Geometric Dimensioning and Tolerancing } \\ \text { MBE } & \text { Model-based Engineering } \\ \text { PDF } & \text { Portable Document Format } \\ \text { PRC } & \text { Product Representation Compact } \\ \text { PLM } & \text { Product Lifecycle Management } \\ \text { PMI } & \text { Product Manufacturing Information } \\ \text { STEP } & \text { Standard for the Exchange of Product Model } \\ & \text { Data } \\ \text { U3D } & \text { Universal 3D } \\ \text { VDA } & \text { German Association of the Automotive } \\ \text { VRML } & \text { Industry }\end{array}$

\section{D MBE AND VISUALIZATION TECHNOLOGY}

3D Model-Based Engineering (MBE) is an approach to product design, manufacturing, and support where a digital three-dimensional representation of the product serves as the normative source for information communicated throughout the product's lifecycle and supply chain. [1] In MBE, the "model" serves as a container not only of nominal geometry information but also of Product Manufacturing Information (PMI) as well as supplementary notes, reports, multimedia, and any other digital data useful for documenting the product.

While products with relatively short lifecycles may not have a need for maintaining data for extended periods of time, the aerospace and defense sectors have vehicle and weapons programs that have lifecycles measured in decades. These industry sectors are also two of the most dependent users of Product Lifecycle Management (PLM) technologies and processes, which by nature promote the use of a 3D modelbased environment and are the most likely to benefit from using it. [2][3]

As an example of a 3D model consider Figure 1, an Adobe Reader $^{1}$ screen shot (downloaded from www.adobe.com/manufacturing/3dpdfsamples/3dsolutions) of a bracket part visualization created from a native Computeraided Design (CAD) model. There are several points worth mentioning about this example.

- The visualization can be rotated and otherwise manipulated to maximize understanding by humans. However, it is not intended for exchange between CAD systems. It complements rather than replaces the original CAD model.

- $\quad$ The image has PMI callouts, using the standard syntax to specify geometric dimensioning and tolerancing (GD\&T) annotations. Even though the visualization lacks the full semantics of the original CAD model, it is helpful in communicating to humans how to manufacture the part arguably more so than an annotated 2D drawing would be.

- The callout at the top of the figure is unstructured text. This is also part of the product data. In fact, product data can include arbitrary attachments such as spreadsheets containing analysis results, video data, and other associated information relevant to the product. These attachments are important and need to be managed and monitored, even if CAD and CAM systems do not "understand" their data formats.

\footnotetext{
${ }^{1}$ Mention of commercial products or services in this paper does not imply approval or endorsement by the authors or their employers, nor does it imply that such products or services are necessarily the best available for the purpose.
} 


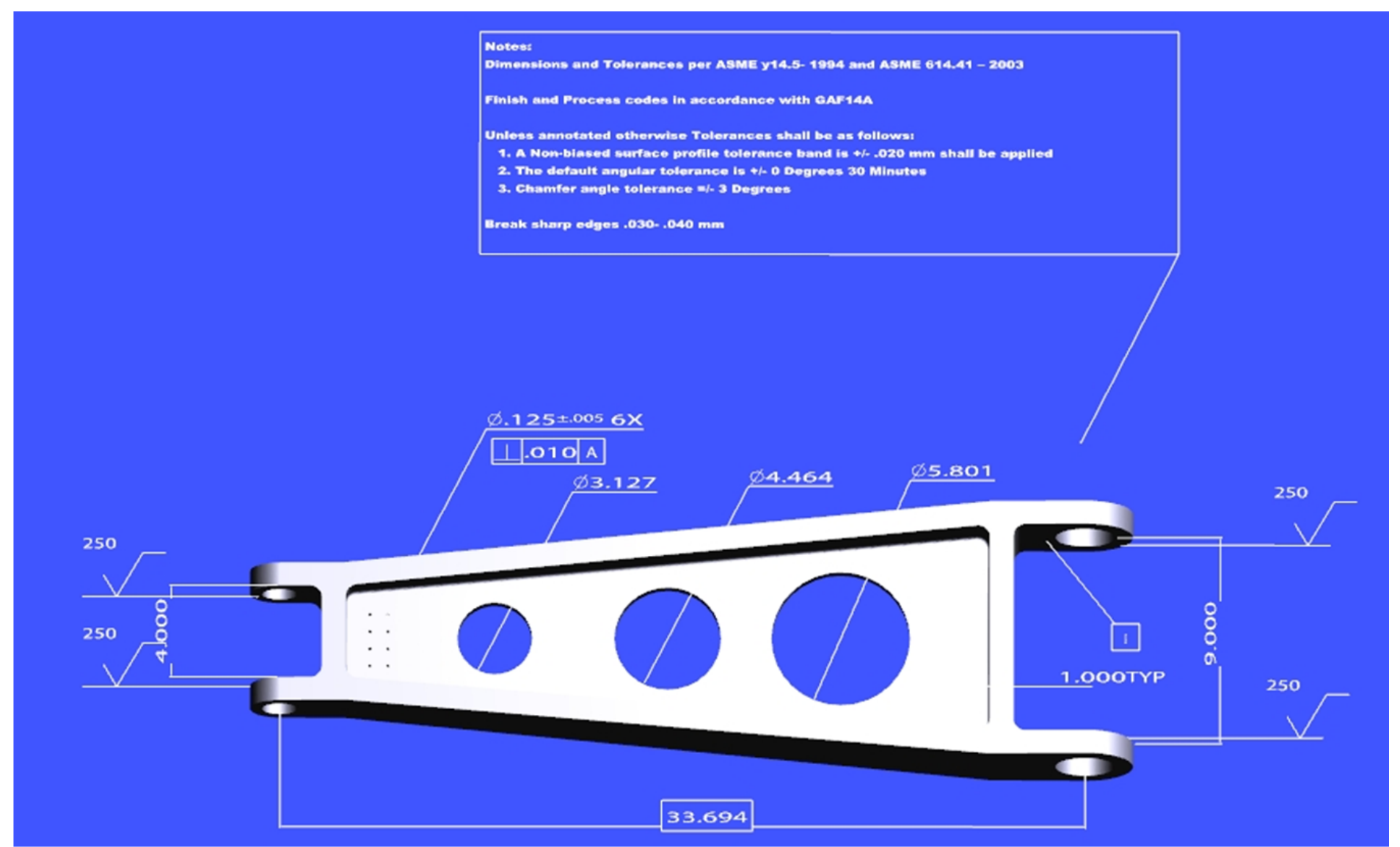

\section{Figure 1. 3D visualization of a bracket part with PMI.}

PMI is a particularly critical constituent of MBE. Loosely defined, PMI can include any sort of information defining a product's components for manufacturing, inspection and sustainment. PMI includes geometric dimensions and tolerances (GD\&T), as well as material specifications and other annotations, specified in a formal language whose syntax and semantics are defined in several American Society of Mechanical Engineers (ASME) and International Organization for Standardization (ISO) standards. PMI is essential to developing complete product data. Manufacturing cannot take place without it. Therefore, MBE requires not only the ability to edit and display PMI, but also the ability to feed GD\&T semantics to computer-aided manufacturing (CAM) systems and other downstream applications. [4]

MBE is appealing to industry for a number of reasons.

- Having a single, normative information source makes data management easier. With the model as master, the model effectively serves as a centralized database such that all information presented to humans or fed to other applications/processes is generated from the model. This is analogous to a report being generated on demand from a database. Thus there is no need to archive or manage this generated information, unless it is directly used to satisfy legal requirements such as type design certification for an aircraft.

- $\quad 3 \mathrm{D}$ is superior to 2D for promoting understanding and ease of searching data. [5][6] This is particularly true for younger engineers, who are accustomed to information being presented as 3D graphics. But there is a caveat. Information overload reduces understandability, so user interfaces must be able to provide for layering and/or selective hiding of the graphics.

- Recent advances in visualization technology (discussed below) and the availability of low-cost software now make it feasible for product designers to share both precise geometry and PMI with suppliers, machine shops, and other collaborators - without requiring that the collaborators buy expensive CAD systems.

- Not only does MBE enable information sharing, but it also supports access control to proprietary or sensitive data. Information may be selectively extracted from the model as needed, depending on the recipient's "need to know." For example a CAM system needs PMI in order to manufacture a part but does not require knowledge of how the design was created. Therefore, it is appropriate that the information sent to the CAM system include precise geometry and PMI but not include any procedural information or parametric data. 
ISO 10303 - also known as STEP (Standard for the Exchange of Product Model Data) - provides a neutral, nonproprietary way to represent 3D geometry and PMI. [7][8][9] STEP is useful in MBE both as a means of exchanging models between CAD systems and as a means of archiving models. But the need to communicate models to people who are not designers and applications other than CAD systems has grown. As a result, visualization technologies such as JT [10] - a data format developed by Siemens PLM Software - and PRC [11] (Product Representation Compact) - an Adobe format for embedding 3D data in a PDF [12] file - are becoming increasingly important. In fact, some visualization file formats are undergoing standardization as discussed later in this paper.

Visualization technology is an enabler for communications in the modern product design, manufacturing and support environments. Historically, the use of drawings for humanconsumable information was the conduit for information exchange. Designers used drawings to communicate design intent, manufacturing engineers used drawings to plan assembly processes, and service and support technicians used drawings to maintain and support a product in the field. As the digital backbone that supports the modern product data enterprise has been propagated throughout the extended product lifecycle, an obvious medium of communication has evolved the lightweight visualization format and its associated viewing technology.

The use of lightweight collaborative formats derived from fully-featured CAD models offers a low-cost way of viewing and consuming geometry and PMI information. Costs are reduced in areas such as the number of full CAD licenses necessary within a corporation, the time spent trying to leverage fully associative CAD models in areas where it is unnecessary, and the reduced time to communicate product information and model metadata throughout the lifecycle given the use of web-based delivery and authoring mechanisms. Through the use of web-based components in the lightweight formats, collaboration is arguably much easier. The file formats are smaller, the viewing utilities work well in the web-based environment, and a sophisticated training regimen is not required to use the visualization formats or their viewers.

In addition to supporting collaboration, these formats make it easier to consume design and manufacturing information in other parts of the lifecycle. Viewing tools are more efficient and use less systems resources than do full CAD platforms, reducing the overall software costs for a corporation and making it easier to implement low cost hardware for those people who do not require as much computing resources. These data formats are also suitable for the mobile computing environment because of their relatively low computing overhead and the inclusion of web-friendly elements within their file definition. Both mobile devices and low-cost network computing technology are used by more people than ever before, which offers companies an opportunity to deploy visualization technology more widely. Another aspect of these formats that promotes collaboration is their robust mathematical definition compared to their relatively small file size. A precise data representation without the impact of large file sizes allows these formats to be used for data archival, manufacturing inspection, supplier collaboration and other areas where moving data efficiently and accurately is important.

\section{VALIDATION AND MODEL CONSISTENCY}

What truly constitutes the 3D product definition? Is it the CAD database only? Is it the model plus drawings? Does it include analysis results? One of the fundamental requirements of a functionally complete MBE environment is that a fullydefined, fully-annotated 3D CAD model be the master source of product definition information. While this may not be a reality today, technology is moving in the direction to enable such an environment, driven by the need for both human and machine consumption of model data. In recent years, leading aerospace and automotive manufacturers have implemented MBE and thereby motivated the technology maturation necessary for other companies to follow suit.

In order to manage a product's lifecycle, one must manage the data lifecycle that accompanies it. One area where this is evident is the format in which product data is shared. In order for MBE to be effective, a digital definition of the product must exist that is correct, complete, and unambiguous. Authorized, equivalent derivative forms of the master data must be generated to support the user's task at hand without losing the embedded sophistication and richness of the original product definition. Nowhere is this more obvious than in the use of lightweight collaborative formats throughout various industry sectors today. While the underlying technology driving these formats has been available for many years, it has only been within the last five years when the use of these data formats has begun to take shape in a way as to predict future direction.

What is meant by "lightweight collaborative format?" According to Ding et. al., [13] lightweight formats arose out of the need for enhanced communication and collaboration between distributed design teams without the overhead associated with using the native CAD models. In addition, viewing and manipulation of non-native CAD data, file size implications of native CAD data, incompatibility between native CAD formats, and the protection of intellectual property are all issues that have given rise to the use of the lightweight collaborative formats in various stages of the product lifecycle. [14][15] While the differences between lightweight collaborative formats can be substantial, the nature of their use is beginning to converge in areas outside of the typical visualization realm. These formats were originally meant to communicate visual information for human consumption, such as part identification or component fit within the larger assembly. This was due in large part to the inherent imprecision of their geometric definition of the object as it was derived from the CAD model. This also allowed the file size of these 
formats to remain relatively low when compared to the native CAD model or to the common STEP format.

However, as the technology providers began to enable more robust and precise geometric definitions of the product within the collaborative formats, users began to implement them in other workflows within the organization and their file size began to grow. This change in use and file size has now led to the collaborative formats being used to exchange data between original equipment manufacturers and suppliers, finished inspection within a manufacturing environment, and the creation of 3D technical publications and data packages to support entire product programs. [16] Using collaborative formats in this way has also increased their file size due to the necessary product geometric definition and metadata that needs to be carried forward, and it has blurred the line between bona fide standard formats and the contemporary collaborative formats (especially as some of these formats are currently undergoing ISO standardization). Industry now finds itself in a scenario where collaborative formats are being used in the same manner as native CAD or STEP formats, necessitating the development and implementation of a data validation scheme to ensure the product data survives the translation process.

Thus product data validation is a critical component of MBE. If the model is to be the master representation of the product, from which all others are derived, then it had better be accurate, precise, and unambiguous. And all derivative representations must have those same characteristics if they are to be trusted when used outside of the engineering context and the native CAD model representation. The risk of different definitions and interpretations of PMI is much higher than for geometry - which is why validation of collaborative formats is receiving more attention within MBE than for older geometryonly processes.

In a drawing-based environment, the drawing itself (particularly when paper-based) contains the relevant product data and serves as a trusted information source for those using it. When drawings are shared, there is typically little worry about information being lost due to translation error in the medium.

MBE, however, provides multiple ways in which to share product data, but - given the slightly different means by which various software tools define geometry - translation between software applications is a challenge. It is these slight differences that account for most (if not all) of our current data translation errors, hence the need for validation. If companies are to maximize benefits from their CAD data assets, they must be able to trust their data as correct, complete and unambiguous for indefinite periods of time regardless of authoring software version or translator used.

\section{OBSERVATIONS AND ISSUES}

To further understand the overall challenges of MBE standardization and validation, we examine them in the context of the following issues that arise in the representation of digital product data.

\section{Parametric and Procedural Information}

Our discussion so far has emphasized integration of engineering design with downstream functions such as manufacturing or field support. A different scenario is when a design needs to be edited in multiple CAD systems. This may be required when design groups use different CAD software collaborate or when, due to business mergers, acquisitions, partnerships, etc., the design authority migrates between dissimilar systems. Both cases require not only the exchange of precise geometry data but also of design intent, i.e., information about the design process itself. Design intent information generated by CAD systems includes procedures used to create shape models, parameters bound to values in the model, constraints between parameters and/or model elements, and features - the shape "building blocks" used to construct the model. Without design intent, a model cannot be easily edited. [17][18] This is because the precise geometry shows only the final result and says nothing about the steps that got you there. A good analogy - given in [17] and illustrated in Figure 2 - is the representation of a chess game. A board diagram is a "precise" model in that it gives a comprehensive view of the current state of the game. But it lacks information regarding the sequence of moves leading up to the current state. In order to refine a design - or fine-tune a chess playing strategy - one needs that procedural information.
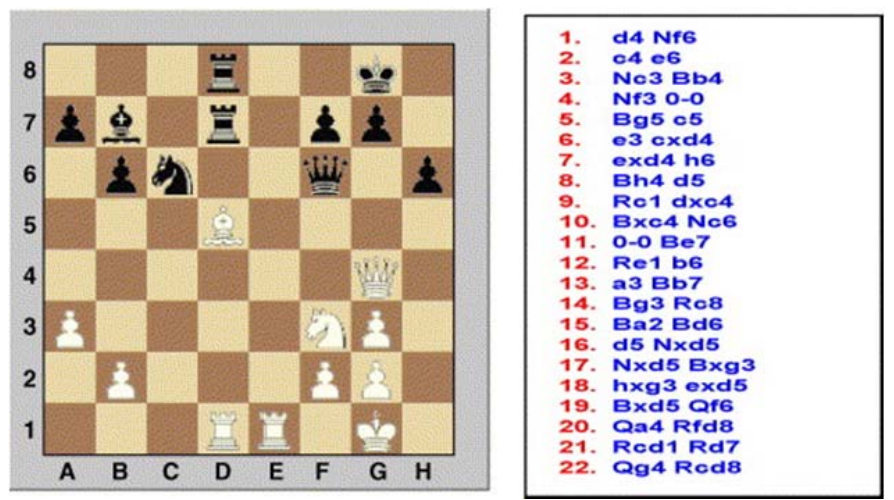

Figure 2. Precise and procedural depictions of a chess game. From [17].

Although CAD systems generate this procedural information, visualization formats do not include it. But, in scenarios where visualization technology is enjoying great popularity, this is a feature and not a bug. Companies often want to share 3D models with partners or customers without giving them the ability to reproduce them in an editable or modifiable form. And CAD vendors can provide tools allowing their customers to convert models to visualizations knowing that their customers cannot use the visualization as a means for migration of their data to a competing software vendor's CAD offering. 
Until recently, no standard existed for exchanging design intent. Today the second edition of STEP AP203 [9] supports some design intent, as does the latest version AP214. [8] But so far no CAD vendors have implemented this functionality.

\section{Document versus Model}

The model as master is a primary tenet of MBE. But sometimes a document-centric metaphor is more useful. For many actors in a product's lifecycle, a written report is the clearest form of communication. Also, MBE may be the ideal but not the reality - an engineering process may require drawings that have yet to be replaced by models. In situation such as these, a paradigm where the document is paramount - and models are attachments to (or embedded in) documents rather than the other way around - may be a better fit. Also, some product definition metadata is best communicated in document form as a complement to the master model definition, rather than forcefitting it into a 3D model format.

The PDF standard (ISO 32000) [12] is particularly well suited for document-centric engineering processes. The PDF format is essentially a packaging mechanism for whatever formats one wants to exchange with collaborators. Thus a PDF instance can include reports, multimedia, drawings, and of course 3D visualizations. For example, the image shown in Figure 1 was captured from a PDF document including video, a spreadsheet, etc. The PDF specification is agnostic regarding which of an instance's constituent parts - if any - is the "master."

\section{Evolving Technology}

MBE technology continues to evolve. CAD/CAM vendors have implemented the first generation of PMI presentation and semantics standards, and the STEP, JT, and PRC specifications are being revised to support exchange of PMI graphics and semantics (structured data). As a result, design systems have a greater ability than ever before to offer information to manufacturing systems, and manufacturing systems are better able to consume that information. However, successful integration of design and manufacturing systems requires not only the existence of exchange standards, but also requires standards for interoperability testing and technology implementing those standards.

The ISO TC213 and ASME working groups responsible for PMI presentation and semantics have recently released new versions of the PMI standards increasing their complexity and representational richness by an order of magnitude. This will drive a new cycle of innovation in CAD/CAM software, which will in turn lead to revisions of CAD data exchange standards to "catch up" with the software vendors.

The evolution of the technology underlying the data formats used in documenting the product lifecycle makes it a challenge to select and implement a specific format. [14][15] It is nearly impossible to know for sure whether the data source and the data destination will remain congruent in perpetuity. In
MBE, the 3D digital product definition is the conduit by which various stages of the product lifecycle communicate with each other. Incompatibility between native CAD data formats makes this communication difficult, leading to the need for validation of 3D product definitions as explained later in this paper.

\section{Supplemental Geometry and Advanced PMI}

ASME Y14.41, a national standard for PMI presentation in 3D models, defines supplemental geometry as "Geometric elements included in product definition data to communicate design requirements but not intended to represent a portion of the manufactured product." [19] Examples of supplemental geometry elements include cutting planes, center lines, and Cartesian points. Supplemental geometry is a critical component of MBE for two reasons. [20]

1. PMI can refer to supplemental geometry elements. Thus, without supplemental geometry, PMI is of limited use.

2. Automated machining and inspection processes require tool target information, which in turn requires supplemental geometry.

Clearly it is important that supplemental geometry be successfully transmitted from CAD models to downstream processes. However, recent results testing model translation between CAD systems using JT as an exchange format [21] are cause for concern. Conducted jointly by the ProSTEP consortium (http://www.prostep.org) and the German Association of the Automotive Industry (VDA http://www.vda.de), the tests indicate a high success rate translating precise geometry and PMI graphics but less success translating supplemental geometry and PMI semantics. As Figure 3 shows, information for human consumption was translated more reliably than information for machine consumption. Another way to interpret the results is that the more "semantic" the information, the less successful the translation.

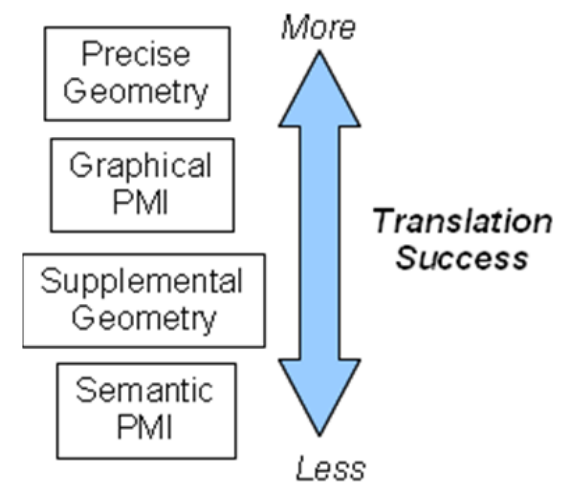

Figure 3. Translation success between CAD systems, using $\mathrm{JT}$ as an intermediary.

The ProSTEP/VDA report does not elaborate on the source of translation problems, i.e., whether the cause is due to JT export from the originating CAD system, JT import to the 
destination CAD system, or issues with the JT format itself. Nevertheless, the report shows areas where improved CAD translation is needed and also shows how the growing usage and capabilities of JT and other lightweight formats add to the challenges of interoperability testing. The primary challenge is to properly organize and document the intent of this information in the master model, and then to accurately translate this into the various derivative formats required for downstream consumption.

\section{Evolving Definition of "PMI"}

Most downstream tools and processes supporting 3D annotations today are simply displaying this data for human consumption as they have been doing with 2D drawings for many years. Thus the technology focus has been on ways to place planar annotation symbology in the space around the 3D model geometry. To avoid the "fur ball" effect (i.e., information overload), authoring and viewing applications support "saved views" (also known as captures, combined states, or model views) defining a camera position, zoom level and visibility of annotation and geometry entities. The human user then activates the display of each saved view to aid in understanding the data.

Some manufacturing and inspection technologies are now being upgraded to directly consume the annotation data directly, without regard for how it is displayed graphically. They require a complete semantic definition of each PMI construct, including each data element and the precise relationships with the model geometry. For example, a geometric tolerance defining the positional variation of a pattern of holes can be visually defined with a few properly placed annotations: a feature control frame and multiplicity text note with leader line to one of the holes and one to three datum feature symbols attached to geometric entities defining the datum reference frame(s). A complete semantic definition requires clear identification of all geometry defining all holes in the pattern, the geometry for all aspects of the datum reference frames with explicit connections to the positional tolerance construct, and a "machine-interpretable" definition of each element (tolerance values, modifiers) which would otherwise be displayed in the feature control frame.

As downstream processes increase their ability to consume PMI semantics, this in turn raises the bar for the level of semantic completeness required in the authoring and translation technologies.

\section{Standardization Issues}

What do we mean by a "standard" in the context of MBE? Such standards may be one of at least these types: [22]

1. Open Standards relate to the general idea of interoperability and integration - an agreement that people make so that products and systems made by different parties can work together. Open standards are not software applications; they are only specifications explaining how information should look. Open standards are developed by consensus in an industry group. There is a tremendous variation in the membership rules and processes for these organizations, and they range from official organizations, such as ISO and ASME to vertical industry groups such as the Strategic Automotive product data Standards Industry Group (http://www.sasig.com). STEP is an example of an open standard. It is developed by ISO, with the help of industrial consortia, such as PDES, Inc. (http://pdesinc.aticorp.org) and ProSTEP.

2. Industry Standards are technologies that are commonly used, but are not open or democratically managed by a group of users. The current JT file format specification is an example of an industry standard. There are a number of companies involved in the JT Open consortium (http://www.jtopen.com) but because one company wields a tremendous amount of control over the process, JT is classified as an industry standard, not an open standard.

Standards address the changes in technology development and usage over time. For MBE, what is primarily at issue is the nature by which CAD software tools encode information when storing it in their native file format and when they export data into alternate formats. Some alternate formats are open standards (e.g., STEP and PDF), while others are industry standards (e.g., JT and PRC). In fact, the former are more like data models or wrappers for holding content than they are a true data format, while the latter are formats provided by a specific technology vendor, thus possessing proprietary characteristics. The proprietary nature of some collaborative formats makes it difficult to build process and other technologies around them due to the uncertainty over the long-term viability of the data.

In addition, those collaborative formats that are transitioning from industry standards to open international standards are taking different paths to get there. The most notable example is Universal 3D (U3D), [23] an underlying geometry representation format for the 3D PDF technology and alternative to PRC, which is passing through the "documentcentric" ISO committee TC171/SC2. The JT format and others are passing through the more "product data-centric" ISO TC184/SC4 committee. While this may seem appropriate, and it may be, it raises a question about how product data is treated in the eyes of the international standards community - is it product data, or are all of these formats simply considered documents generated by the product lifecycle processes? The answer to that question has operational impacts both for technology providers and for companies that build their processes around this technology. A split in how these formats are handled within the larger international community could have impacts on how companies build processes around their use. In addition, it raises the question as to whether the notion of a document is comprehensive or specific enough to encompass all the requisite information generated during the product lifecycle. 
Another issue regarding the progression of collaborative file formats from industry standards to open standards is whether the collaborative file formats as currently specified are "open" enough to meet the requirements of standards development organizations. A collaborative format may be optimized for a particular vendor's software platform, at the expense of interoperability with competing vendors' products. A benefit to having a format being recognized as an open standard is that specification of the format has been vetted by a committee where no single member has inordinate influence at least in theory, thus giving users of the format some assurance that their validation tools and processes will remain viable. A key point to be mindful of is that most people in this data exchange and validation space see the ownership of a data format by a specific company as potentially detrimental with regard to long-term archival and accessibility of data encoded into such a format. Whereas, the maintenance (and long-term development and propagation) of a format by an international standards body is seen as a bit more palatable.

\section{LOOKING AHEAD}

As we look ahead to the future of communications within the product lifecycle, it is clear that the CAD model is changing from its historical role of documenting geometric definition to being the conduit for information exchange within multiple industrial processes and workflows. Technology providers are developing product definition tools beyond the scope of geometry to include textual and symbolic data that in the past would have been communicated via 2D drawings. As such, the user community must adapt its ways of working and knowing relative to this technology, while facing new standardization and validation complexities brought on by this brave new world.

Product definition information taking a visual (or geometric) form is typically easy for a human to consume through the use of accepted practices and standard definitions of format. Older visualization technologies such as the Virtual Reality Modeling Language (VRML) [24] presented this information in a way that made this relatively easy to accomplish; however, they lacked much of the richness of meaning that today's current and emerging product definition forms possess. The new formats being created approach communications from the point of representation, allowing for the possibility of product definition information to be not only human-consumable but also machine-consumable.

However, clear and unambiguous encoding of the representation's underlying semantics is a challenge. This is one of the driving reasons why process and data validation is extremely important in an MBE environment. Practical and effective validation must incorporate all types of digital product data and all formats the data may take throughout the product lifecycle. Companies must identify the key characteristics required to remain consistent while allowing other aspects of the digital definition to vary within acceptable limits, thus accommodating the inevitable differences between MBE technologies.

As derivative formats have emerged from the native CAD dataset, they have transitioned from being used for visualization and simple communication between humans to being part of the process in long-term archival, machining operations, and quality control and inspection. They are also being used in marketing pieces and virtual environments where the native CAD database would have been used previously. These new uses often result in lower costs, increased geometric accuracy, and better communications for the users, but not without quality assurance of the translation between CAD systems and derivative formats.

\section{REFERENCES}

[1] " 5 Secrets for Making the Model-Based Enterprise a Reality.” White paper from Renaissance Services. 2008. http://www.mbesecrets.com/

[2] Schmitt, P. (2007). PLM in aerospace: High-profile projects help extend PLM technology through the entire aerospace supply chain. Manufacturing Engineering, 138(3). Retrieved from http://www.sme.org/cgi-bin/findarticles.pl?\&ME07ART16\&ME\&20070311\&\&SME\& on January 18,2011.

[3] Van Wijk, D. et. al. (2009). Integrated design and PLM applications in aeronautics product development. Proceedings of the $19^{\text {th }}$ Annual CIRP Conference, Cranfield University, UK, March 30-31, 2009.

[4] V. Srinivasan. "STEP in the Context of Product Data Management.” In Advanced Design and Manufacturing Based on STEP. Xun Xu and Andrew Y.C. Nee eds. Springer London. doi: 10.1007/978-1-84882-739-4. 2009. Pages 353-381.

[5] Theodore J. Branoff, Nathan W. Hartman, and Eric N. Wiebe. "Constraint-Based, Three-Dimensional Solid Modeling in an Introductory Engineering Graphics Course: Re-examining the Curriculum.” Engineering Design Graphics Journal. Volume 66. Number 1. Winter 2002. Pages 5-10.

[6] S. K. Ong, M. A. Mannan, "Virtual reality simulations and animations in a web-based interactive manufacturing engineering module," Computers \& Education, Volume 43, Issue 4, December 2004, Pages 361-382.

[7] ISO 10303-1. Industrial automation systems and integration - Product data representation and exchange Part 1: Overview and fundamental principles. First edition 1994-12-15.

[8] ISO 10303-214:2010. Industrial automation systems and integration - Product data representation and exchange Part 214: Application protocol: Core data for automotive mechanical design processes.

[9] ISO 10303-203:2011. Industrial automation systems and integration - Product data representation and exchange Part 203: Application protocol: Configuration controlled 3D design of mechanical parts and assemblies. Edition 2. 
[10] JT File Format Reference. Version 9.5. Rev-A. Siemens Product Lifecycle Management Software Inc. 2010.

[11] PRC Format Specification. Version 8137. Adobe Systems Incorporated. 2008.

[12] ISO 32000-1:2008. Document management - Portable document format - Part 1: PDF 1.7.

[13] L. Ding, A. Ball, J. Matthews, C. McMahon, and M. Patel, "Product representation in lightweight formats for product lifecycle management (PLM),” presented at Fourth Int. Conf. on Digital Enterprise Technol., Bath, UK, Sept. 1921, 2007.

[14] N.W. Hartman and M.A. Lim. (2008). Examining neutral formats for visualization and data exchange," in Proc. of the 2008 IAJC-IJME Int. Conf., Nashville, TN, Nov. 1819, 2008.

[15] N.W. Hartman (2009). Evaluating lightweight 3D graphics formats for product visualization and data exchange. Journal of Applied Science \& Engineering Technology, Vol 3 , Retrieved from https://library.rit.edu/oajournals/index.php/jaset/article/vie $\mathrm{w} / 234 / 0$.

[16] A. Ball, L. Ding, and M. Patel. (2007). Lightweight formats for product model data exchange and preservation. Presented at PV 2007 Conference, Oberpfaffenhofen, Germany, Oct. 9-11, 2007.

[17] Michael J. Pratt, Bill D. Anderson, Tony Ranger. “Towards the standardized exchange of parameterized feature-based
CAD models.” Computer-Aided Design. Volume 37. Issue 12. October 2005. Pages 1251-1265. ISSN 0010-4485. DOI: 10.1016/j.cad.2004.12.005.

[18] Junhwan Kim, Michael J. Pratt, Raj G. Iyer, Ram D. Sriram, Standardized data exchange of CAD models with design intent, Computer-Aided Design, Volume 40, Issue 7, Current State and Future of Product Data Technologies (PDT), July 2008, Pages 760-777, ISSN 0010-4485, DOI: 10.1016/j.cad.2007.06.014.

[19] ASME Y14.41, Digital product definition data practices, The American Society of Mechanical Engineers (2003).

[20] Yochen Boy, Phil Rosche. "Recommended Practices for Supplemental Geometry.” CAx Implementor Forum. November 1, 2010. http://cax-if.org

[21] ProSTEP iViP/VDA. "JT Translator Benchmark." Short Report. Edition 1. February 2010. http://www.prostep.org

[22] Russell S. Peak, Joshua Lubell, Vijay Srinivasan, and Stephen C. Waterbury. "STEP, XML, and UML: Complementary Technologies.” J. Comput. Inf. Sci. Eng. 4, 379 (2004). DOI:10.1115/1.1818683

[23] Ecma International. Standard ECMA-363. Universal 3D File Format. $4^{\text {th }}$ Edition. June 2007.

[24] ISO/IEC 14772-1:1997. Information technology Computer graphics and image processing - The Virtual Reality Modeling Language (VRML) - Part 1: Functional specification and UTF-8 encoding 